\title{
Endoluminal Negative Pressure Wound Therapy (E-NPWT) for anastomotic leakage after rectal resection
}

\author{
Norbert Runkel, $M D$, Ph.D., Mechthilde Birk
}

\begin{abstract}
Background: Local management of anastomotic leakage after rectal cancer resection has traditionally consisted of rinsing and drainage. Transanal endoluminal application of NPWT (E-NPWT or endo-VAC or endo-sponge) is an interesting novel concept that has been explored in a few studies and case series over the last decade. We report herein our institutional experience over a three year period during which E-NPWT was the local treatment of choice for all cases with anastomotic leakage after rectal resection.

Patients and Methods: This study retrospectively evaluated the clinical charts of 147 consecutive patients who underwent anterior rectal resection from 2011-2013. A postoperative anastomotic leak occurred in eight $(5 \%)$ patients from two straight anastomoses, one side-to-end construction, and five colonic pouches. All patients had undergone curative anterior resection with diverting ostomy. Transanal debridement and application of a trimmed foam were performed by rigid or fexibel endoscopy and started without delay after confirmation of diagnosis in seven patients 8-15 days postoperatively. In one patient E-NPWT began after failed fibrin-glue treatment 6 weeks later. The foams were connected to a standard vacuum pump with the pressure set at $-70 \mathrm{mmHg}$ in a continuous mode. E-NPWT was maintained for a median period of $10(5-25)$ days. The treatment intervals were typically 2-3 days.
\end{abstract}

Results: Complete healing was achieved in three cases during E-NPWT. In the remaining five patients, the defect reduced in size to allow further spontaneous healing. This occurred twice. One persistent sinus was successfully sealed with fibrin glue. One persisting recto-vaginal fistula required surgical closure. One large defect secondary to ischemic pouch necrosis required secondary pouch explantation and permanent colostomy. The overall ileostomy reversal rate was $75 \%$.

Conclusion: E-NPWT is feasible and without severe side effects. Early initiation prevents septic progression and results in a high closure rate in patients with pelvic leakage. Although complete healing is not achieved in recto-vaginal fistulas or ischemic necrosis of the neorectum, E-NPWT may play a potential role in bridging and damage control.

Keywords-NPWT, Endo-VAC, Endo-Sponge, anastomotic leakage, rectal cancer, postoperative complication

\section{INTRODUCTION}

A NASTOMOTIC Anastomotic dehiscence is associated with an increased early and late morbidity and mortality

Manuscript received 9.2014; revised 10.2014. No conflict of interest declared

Author affiliations: Department of General Surgery, Schwarzwald-Baar Klinikum, Teaching Hospital University of Freiburg, Klinikstrasse 11, 78052, Villingen-Schwenningen, Germany, (NR, MB)

*Correspondence to: Prof. Dr. Norbert Runkel (e-mail: norbert.runkel@ sbkvs.de). causing re-interventions, prolonged hospitalization, impairment of anorectal function, and a delay of adjuvant chemotherapy and stoma reversal. The non-operative management of leakages has recently undergone a fundamental change through the endoscopic adoption of modern concepts of negative pressure wound therapy (NPWT). The novel transanal endoluminal application of NPWT (E-NPWT) has been published in case reports 12 and small series from central Europe ${ }^{3 / 11}$ The potentials and limitations of E-NPWT remain largely unknown to the colorectal community despite the availability of a commercial kit (Endo-SPONGE ${ }^{\circledR}$, B. Braun Melsungen, Germany). The aim of this study was to analyze our results of E-NPWT in a non-selected consecutive group of patients over a 3-year period. We had been using E-NPWT routinely for managing all leaks in the pelvic cavity for some time.

\section{PAtients AND Methods}

\section{A. Patients}

This study analyzed a three-year period since 2011 when E-NPWT had become the first-line treatment for patients with symptomatic leaks after rectal resection with an extraperitoneal anastomosis. A total of 161 patients with rectal cancer underwent abdomino-perineal $(n=14)$ or anterior rectal resection with either partial (PME; $n=30$ ) or total mesorectal resection (TME; $n=107$ ) between $1 / 2011$ and 12/2013.

The rate of laparoscopic surgery was 95\% without any conversion. Sixteen patients with PME received a double stapled end-to-end colo-rectostomy (straight anastomosis), and 14 patients with PME had no anastomosis (Hartmann's situation). The neorectum after TME were constructed as a colon-pouch in 96 patients, a side-to-end reconstruction in 11 patients, and a straight anastomosis in one patient. The anastomoses after TME were double stapled or hand sewn anastomoses in 70 and seven patients, respectively. The anastomoses were protected by enterostomies in 39\% of patients with PME and in $99 \%$ of patients with TME. The anastomoses were not routinely controlled in the early postoperative period in an asymptomatic patient. In case of clinical suspicion of an anastomotic leak, however, CT-scan with transanal contrast medium and endoscopy were performed without delay. The integrity of the anastomosis was always confirmed prior to stoma reversal at a later stage. 


\section{B. Technique}

The technique is similar to that described in detail by Glitsch et al. (5). In summary, endoscopy is performed using flexible scopes or, preferentially rigid instruments. The cavity is entered through the anastomotic defect, which is widened when necessary. Clearing the infectious cavity from debris and generous rinsing with saline are mandatory procedures each time of re-intervention. A polyurethane foam-based NPWT system (RENASYS GO ${ }^{\mathrm{TM}}$, Smith and Nephew Healthcare, Hull, United Kingdom) is used. The pressure level is set at $-70 \mathrm{mmHg}$. The foam is trimmed according to the size and shape of the cavity, so that it can fill out the entire lumen of the wound. The following foams are consequently reduced in size to foster the collapse of the cavity. The sponge is placed inside the cavity or, when downsizing occurs, partially or totally within the rectal lumen. This is why fecal deviation is considered mandatory in our institution.

\section{RESULTS}

Postoperative anastomotic leakage was detected in eight patients (Table I). Anastomotic leakage was clinically identified in six five male and two female patients (total leakage rate $5,0 \%$ ), seven of whom had undergone TME (TME leakage rate $6,5 \%$ ) and one PME (PME leakage rate 3,3\%). Leakage occurred in five colon-pouches, one side-to-end construction, and two straight anastomoses. All but two hand-sewn intraanal anastomoses had been double-stapled. All neorectums has been protected by an ileostomy. Neoadjuvant therapy had been given to five of eight patients prior to resection.

Leakage was associated with severe sepsis in two cases (patient No. 1, 2) one of whom (patient No. 1, TME and colon-pouch) rapidly developed sepsis with multiorgan failure (MODS) caused by peritonitis ascending from a pinhead sized leak between the linear stapler line at the end of colonic stump. This patient required emergent re-laparoscopy and lavage in addition to E-NPWT. The anastomotic defect closed within 10 days, however, recovery from MODS was much prolonged. The ileostomy was never taken down because of anal sphincter insufficiency and systemic tumor disease.

Three were four small leaks $(<1 \mathrm{~cm})$, one midsize leak $(<2$ $\mathrm{cm})$ and three major leaks ( $>1 / 3$ circumference), one of which was secondary to partial ischemic necrosis of the neorectum (patient No. 6, TME with straight anatomosis).

Diagnosis of insufficiency was established after a median of 8.5 days (range 2-18). Endoscopic management with E-NPWT was initiated without delay in seven patients and after 6 weeks in one patient (No. 4) in whom fibrin-sealing had been tried without success. E-NPWT was maintained for a median period of 10 (5-25) days. The endo-sponge was typically exchanged in 2-3 day intervals. The intervention was performed under general anesthesia in the case of sepsis (No. 1), who had abdominal revision simultaneously, and under surgeon-guided Propofol-sedation in four patients, and without any in three.

The leakage/cavity completely healed during E-NPWT in three cases. In the remaining five patients, the defect became clean and small enough to allow further spontaneous healing. This occurred twice. One persistent sinus was successfully sealed with fibrin glue (patient No. 4). The ileostomy was subsequently taken down in five patients. The ileostomy remained in place in one patient due to sphincter insufficiency and progressive tumor disease. In one female patient (No. 7), a recto-vaginal fistula persisted despite reduction in size. She underwent re-laparoscopy, omental interposition and transanal/transvaginal suture. Her ostomy was finally taken down 3 months later. Following restauration of continuity, overall bowel function and defecation were not apparently different from the normal range after anterior rectal resection.

The patient with pouch-necrosis (patient No. 6) required subsequent operative pouch removal with permanent colostomy, because his defect remained significant despite 25 days of E-NPWT.

\section{DISCUSSION}

Anastomotic leakage remains the most devastating complication after low rectal resection. Numerous published studies have analyzed risk factors and protective measures such as diverting ileostomy, however there is little science regarding clinical management of leakage. Most of the knowledge and measures that are being passed down from surgical leaders to their trainees are experience- rather than evidence-based. This includes operative revisions for patients with sepsis and conservative treatment by rinsing and drainage of the leakage cavity in patients without peritonitis. Internally applied NPWT is a new non-operative treatment modality first described by Weidenhagen et al. from Munich in 2003 for active rather than passive drainage 3 The authors have named the technique endoscopic vacuum-assisted closure of anastomotic leakage but another group from Germany has proposed the term endoscopic transanal vacuum-assisted rectal drainage (ETVARD)! 5

We believe that both terms do not precisely describe the potential role of negative pressure therapy. It pursues four principle goals: firstly, effective suction drainage inside the septic cavity with negative pressure transported to the foamtissue contact zone; secondly interruption of continuous contamination of the cavity by colonic mucous and stool; thirdly reduction of the size of the cavity, and lastly healing by promoting granulation. We therefore prefer the term endoluminal negative-pressure wound therapy (E-NPWT). NPWT is a generic name and is not associated with a particular company such as Endo-VAC (V.A.C. ${ }^{\text {TM }}$ KCI, San Antonio, TX, USA) or Endo-SPONGE ${ }^{\mathrm{TM}}$ (B. Braun Melsungen, Germany).

The basic feature of this method is the placement of an open-cell foam into the abscess cavity of the anastomotic leakage and/or into the rectal lumen. The suction tube fixed to the sponge is placed transanally and connected to a vacuum pump. The commercially available kit (Endo-SPONGE ${ }^{\mathrm{TM}}, \mathrm{B}$. Braun, Melsungen, Germany) facilitates the introduction of the sponge with the help of a pusher and overtube. But many surgeons use self-made devices on the basis of off-label use of clinically established foam-based systems just as we did. 


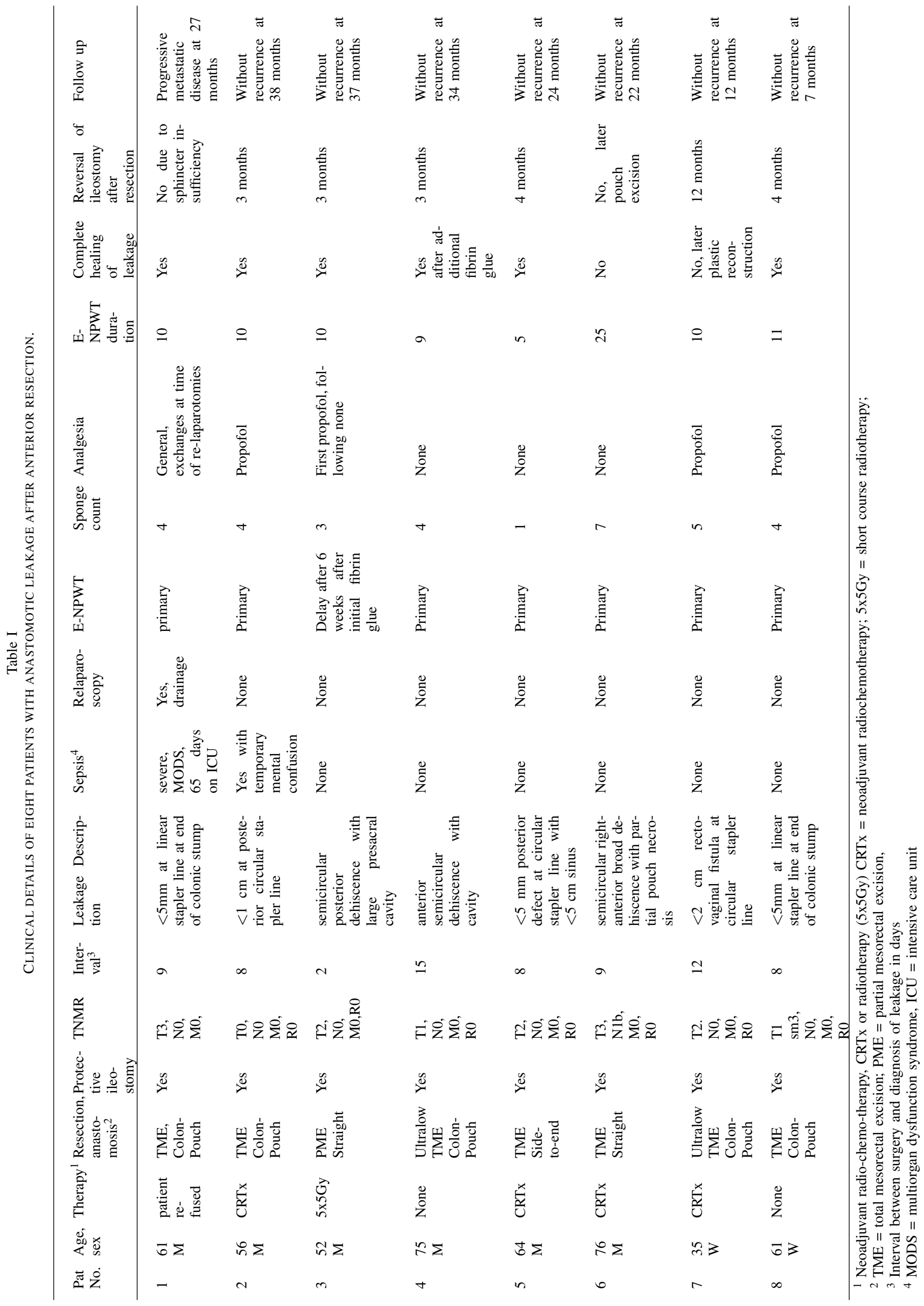


This minimally invasive method is applicable by rigid or flexible endoscopy. If required propofol anesthesia is a comfortable adjunct to achieve painless debridements and exchanges of the sponges. It is our impression that pain reduces with progressive control of pelvic sepsis.

Most technical details of E-NPWT have been adopted from standard negative pressure therapy for acute wounds and open abdomen. The method resembles evidence-based recommendations for acute wound $\mathrm{s}$ and open abdomen developed by an international expert panel chaired by the treatment.principle author of this study (N.R.).12 14 Repetitive endoscopic debridements remain the cornerstones with intervals of revision chosen as required. NPWT is not a substitute for thorough or repeated surgical debridement. Latter should be performed prior to placement of the NPWT dressing. Application of NPWT to cavities containing necrotic tissue is contraindicated. Intervals of re-intervention should not be longer than 3-5 days to prevent granulation ingrowth into the sponge cells. Foam rather than gauze has been the woundfiller of choice in our study and all other publications. Similarly, the use of a protective interface has not been reported. It is important to emphasize than E-NPWT should be continued until healing is complete or until stagnation is observed. An alternative treatment protocol must be considered in the absence of further progress of healing. If the remaining sinus is minute, spontaneous closure will occur without further intervention in the presence of an enterostomy.

A wide range of negative pressures have been reported up to $125 \mathrm{mmHg}, 4$ however no studies have investigated the most appropriate level of negative pressure. Surprisingly, even higher levels were applied with the use of high-vacuum wound drainage systems (B Braun, Melsungen Germany, Redovac $\mathrm{R} 400 \mathrm{ml}){ }^{7}$ In contrast, we recommend pressure levels of less than $-80 \mathrm{mmHg}$ and as low as $-50 \mathrm{mmHg}$ in vulnerable patients (e.g. those with sepsis). The prevalent untested assumption is that the higher the pressure levels, the higher the potential for damage to the surrounding structures. This is in accordance with recommendation for temporary closure of open abdomen with NPWT ${ }^{14}$ There has not been a single incidence of any damage secondary to E-NPWT in the literature. In our and in all other cited papers the pressure was applied in a continuous mode. Intermittent pressure (where pressure settings fluctuate between on and off) or variable pressure levels (where pressure setting fluctuate between high and low) have not been explored for endoluminal therapy.

We strictly used E-NPWT within the hospital setting as a consequence of reimbursement policies in Germany, however, selected patients can be managed on an outpatient basis as was demonstrated in the Netherlands 11

Few series have been published over the last ten years since the first abstract communication by Weidenhagen et al. 3 from Munich, Germany in 2003. Nagell and Holte ${ }^{4}$ from Copenhagen, Denmark reported a small group of four patients with anastomotic leakage after rectal resection with protective ileostomy. E-NPWT treatment was immediately commenced at the time of diagnosis of insufficiency. One patient was lost following cerebral hemorrhage. The three remaining patients were treated with transrectal vacuum for a median of 13-37 days and completely healed within 43, 51, and 19 days. The control group of six patients healed after 336days $(52-1,464)$.

The Munich group ${ }^{6}$ described a series of 29 patients treated with endo-sponge. Most patients underwent surgery for cancer including nine of them with neoadjuvant radiochemotherapy. Definitive closure was achieved in all but one patient $(97 \%)$. Treatment lasted 34 days on average (range 4-79 days) and the mean number of sponge exchanges was 11 (range 1-27). Stoma reversal rate was 22 out of the 25 patients with a protecting stoma.

Mees et al. 7 from Muenster, Germany, examined a small series of 10 patients with a presacral abscess following anastomotic leakage after low anterior resection or restorative proctocolectomy. E-NPWT was compared with traditional management (five patients in each group). Negative pressure was applied for a median time of 27 days without specific complications. Wound healing was significantly faster with E-NPWT compared to traditional management. Hospital stay was slightly shortened by E-NPWT. Median time for wound closure was 45days (range 32-68 days) compared to 101 days (range 79-125 days) in the control group $(\mathrm{p}<0.001)$.

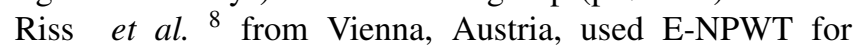
anastomotic disruption (6 patients) and rectal stump disruption (3 patients). Median time of sponge treatment was 3 weeks (2-8 weeks). Healing was induced in $6(66 \%)$ patients. Treatment failure occurred in patients with near total anastomotic disruption. Pain during E-NPWT was low (median 3, range 0-6 according to the visual analogue scale VAS of $0-10$.

The long-term efficacy of initially successful endo-sponge assisted therapy was assessed in another paper from the same group. 10] Twenty patients were recruited from six surgical departments in Vienna. The median follow-up duration was 17 months (1.5-29.8). Five patients (25\%) developed a recurrent abscess. Median time between last day of endo-sponge therapy and occurrence of recurrent abscess was 255 days (21-733). One of these patients was treated by computed tomographyguided drainage, and in 3 patients Hartmann's procedure had to be performed. One patient had only minimal clinical signs and further therapy was still under discussion at the time of publication. The authors speculated that late onset of anastomotic leakage was associated with a tendency for a high probability of recurrent abscess formation

Glitsch et al. ${ }^{[5]}$ from Greifswald, Germany, treated a total of 17 patients with anastomotic leakage after resection of the rectum or rectosigmoid colon with instant endoscopic debridement and endoscopic placement of foam into the cavity. The mean duration of therapy was 21 days, with a mean of 5 sponge exchanges and 11 endoscopies. Treatment was successful in 16 patients. The mean total time to closure of the cavity was 53 days. One patient required a Hartmann's procedure.

A second paper from the same group prospectively investigated the impact of neoadjuvant radiochemotherapy on endoscopic transanal vacuum-assisted rectal drainage ${ }^{[9}$ E-NPWT was performed as first-line treatment in 26 rectal cancer patients including 14 following neoadjuvant radiochemotherapy. In 23 of 26 patients, E-NPWT was successfully completed. Radiochemotherapy was significantly associated with larger size 
of leakage cavity, longer treatment duration, higher frequency of endoscopies and sponge exchanges, and prolonged closure time.

A Dutch multicenter observational E-NPWT study included 16 patients with leakage after surgery for rectal cancer $(n=$ 13) or ulcerative colitis $(n=3) ![1]$ The total closure rate was $56 \%$ ). Of the 16 patients, eight patients started with the endosponge treatment within 6 weeks (13-39 days) after the initial surgery. In the remaining eight patients treatment was started later than 6 weeks (43-1,602 days) after surgery. The closure rate was $75 \%$ in the early treatment group vs. $38 \%$ in the late treatment group $(p=0.315)$. Closure was achieved in a median of 40 (range 28-90) days with a median number of 13 sponge replacements (range $8-17$ ).

In our present study on a 3-year series of non-selected consecutive patients with leakage after anterior resection for rectal cancer, the closure rate without further surgery was $75 \%$ (6/8 patients). The closure rate increased to $88 \%(7 / 8$ patients) when the patient with additional surgical closure of a recto-vaginal fistula was included. All patients had a primary diverting ostomy which we consider mandatory for E-NPWT. The principle of fecal deviation contributed to our low overall leakage rate of $5 \%$ after rectal resections.

Until now, there is insufficient literature to determine whether E-NPWT is superior to wait-and-see policy under fecal diversion. But a few messages from the available evidence can be extracted. Firstly, E-NPWT is technically feasible without complications associated with this treatment. Secondly, some principles of care from NPWT for external wounds can be adopted for internal wounds: the priority of debridement, active suction at the contact zone of sponge and tissue, the harmlessness of negative pressure less than $-100 \mathrm{mmHg}$, the time intervals and the search for other modalities when further progress is lacking, a high level of expertise and hospital resources required. The effects of E-NPWT resemble those of NPWT for external septic wounds, too: rapid re-direction of septic material, sealing in the early phase and promotion of granulation tissue with reduction of the cavity size in the later phase. Closure of the leakage whole/fistula opening can be expected in the great majority of patients within 3-6 weeks. ENPWT is well tolerated by the patients with minimal analgesic requirements between the intervals of sponge insertion and maximal mobility.

The third massage from the available evidence regards limitations of E-NPWT. One negative prognostic factor is preoperative radiochemotherapy. ${ }^{9}$ The timing of the initiation of the treatment plays an important role. Early onset within 6 weeks of surgery resulted in a closure rate of $75 \%$ compared to a $38 \%$ closure rate in patients that started later $(p=0.315)$.11 The overall closure rate of the Dutch trial $(56 \%))^{11}$ was lower than that achieved in Munich $(97 \%)^{\sqrt[6]{6}}$ or in our institution (75\%). The Dutch explanation seems plausible that the progressive development of fibrosis of and around the neorectum precludes alignment of the bowel with the abscess cavity. We therefore recommend to initiate E-NPWT without delay once the diagnosis of leakage is confirmed. This emphasizes the need for instant access to flexible and or rigid endoscopy.

Another important limitation of E-NPWT is the complexity and size of the anastomotic defects. Leakages secondary to circumscribed stable line disruption are good candidates for complete healing. Subtotal dehiscence or multiple fistulas 8 large cavities 5 may not be closed by this technique. We have identified necrosis as another cause of failure. When leakage is a result from ischemic wall necrosis of the neorectum as observed, closure cannot be achieved. Under these circumstances the value of E-NPWT is limited to damage control and to bridge for later definitive surgical excision of the neorectum. A re-construction of a neorectum was not considered in our patient.

Finally, many questions regarding selection of patients and technical details of application remain to be answered such as the comparison of commercial versus self-made kits. Future studies should examine the long term outcome and function after successful treatment with E-NPWT and the necessity of diverting the fecal stream. Studies should explore the potentials of E-NPWT in intraperitoneal leaks after colon resection and for prophylactic perioperative use.

\section{CONCLUSION}

Our study adds another piece of evidence to the literature regarding the management of anastomotic leakages after rectal resection. E-NPWT is feasible and without severe side effects. Onset should be early or immediate after diagnosis. Closure of the cavity and distracted anastomosis can be achieved in high numbers within several weeks facilitating the take down of the protective ostomy at a later stage. E-NPWT may help reversing sepsis in pouch necrosis but fails to induce complete healing in this situation.

\section{REFERENCES}

[1] J. Einenkel, B. Holler, and A. Hoffmeister, "Sonographic diagnosis and endo-SPONGE assisted vacuum therapy of anastomotic leakage following posterior pelvic exenteration for ovarian cancer without using a protective stoma," J Gynecol Oncol, vol. 22, no. 2, p. 131, 2011. [Online]. Available: http://dx.doi.org/10.3802/jgo.2011.22.2.131

[2] P. Van Koperen, M. Henegouwen, J. Slors, and W. Bemelman, "Endo-sponge treatment of anastomotic leakage after ileo-anal pouch anastomosis: report of two cases," Colorectal Disease, vol. 10, no. 9, pp. 943-944, 2008. [Online]. Available: http: //dx.doi.org/10.1111/j.1463-1318.2008.01485.x

[3] R. Weidenhagen, K. U. Gruetzner, T. Wiecken, F. Spelsberg, and K.W. Jauch, "Endoscopic vacuum-assisted closure of anastomotic leakage following anterior resection of the rectum: a new method," Surgical endoscopy, vol. 22, no. 8, pp. 1818-1825, 2008.

[4] C. F. Nagell and K. Holte, "Treatment of anastomotic leakage after rectal resection with transrectal vacuum-assisted drainage (VAC)," International Journal of Colorectal Disease, vol. 21, no. 7, pp. 657-660, jan 2006. [Online]. Available: http://dx.doi.org/10.1007/ s00384-005-0083-4

[5] A. Glitsch, W. von Bernstorff, U. Seltrecht, I. Partecke, H. Paul, and C. Heidecke, "Endoscopic transanal vacuum-assisted rectal drainage (ETVARD): an optimized therapy for major leaks from extraperitoneal rectal anastomoses," Endoscopy, vol. 40, no. 3, pp. 192-199, mar 2008. [Online]. Available: http://dx.doi.org/10.1055/s-2007-995384

[6] R. Weidenhagen, K. U. Gruetzner, T. Wiecken, F. Spelsberg, and K.-W. Jauch, "Endoscopic vacuum-assisted closure of anastomotic leakage following anterior resection of the rectum: a new method," Surg Endosc, vol. 22, no. 8, pp. 1818-1825, dec 2007. [Online]. Available: http://dx.doi.org/10.1007/s00464-007-9706-x

[7] S. T. Mees, D. Palmes, R. Mennigen, N. Senninger, J. Haier, and M. Bruewer, "Endo-vacuum assisted closure treatment for rectal anastomotic insufficiency," Diseases of the Colon \& Rectum, vol. 51, no. 4, pp. 404-410, 2008. [Online]. Available: http: //dx.doi.org/10.1007/s10350-007-9141-z 
[8] S. RISS, A. STIFT, M. MEIER, E. HAIDEN, T. GRÜNBERGER, and M. BERGMANN, "Endo-sponge assisted treatment of anastomotic leakage following colorectal surgery," Colorectal Disease, apr 2009 [Online]. Available: http://dx.doi.org/10.1111/j.1463-1318.2009.01885.x

[9] W. von Bernstorff, A. Glitsch, A. Schreiber, L. I. Partecke, and C. D. Heidecke, "ETVARD (endoscopic transanal vacuumassisted rectal drainage) leads to complete but delayed closure of extraperitoneal rectal anastomotic leakage cavities following neoadjuvant radiochemotherapy," International Journal of Colorectal Disease, vol. 24, no. 7, pp. 819-825, feb 2009. [Online]. Available: http://dx.doi.org/10.1007/s00384-009-0673-7

[10] S. Riss, "Recurrent abscess after primary successful endo-sponge treatment of anastomotic leakage following rectal surgery," WJG, vol. 16, no. 36, p. 4570, 2010. [Online]. Available: http://dx.doi.org/10. 3748/wjg.v16.i36.4570

[11] P. J. van Koperen, M. I. van Berge Henegouwen, C. Rosman C. M. Bakker, P. Heres, J. F. M. Slors, and W. A. Bemelman, "The dutch multicenter experience of the endo-sponge treatment for anastomotic leakage after colorectal surgery," Surg Endosc, vol. 23, no. 6, pp. 1379-1383, nov 2008. [Online]. Available: http://dx.doi.org/10.1007/s00464-008-0186-4

[12] H. Birke-Sorensen, M. Malmsjo, P. Rome, D. Hudson, E. Krug, L. Berg, A. Bruhin, C. Caravaggi, M. Chariker, M. Depoorter, C. Dowsett, R. Dunn, F. Duteille, F. Ferreira, J. F. Martínez, G. Grudzien, S. Ichioka, R. Ingemansson, S. Jeffery, C. Lee, S. Vig, N. Runkel, R. Martin, and J. Smith, "Evidence-based recommendations for negative pressure wound therapy: Treatment variables (pressure levels, wound filler and contact layer) - steps towards an international consensus," Journal of Plastic, Reconstructive \& Aesthetic Surgery, vol. 64, pp. S1-S16, sep 2011. [Online]. Available: http://dx.doi.org/10.1016/j.bjps.2011.06.001

[13] E. Krug, L. Berg, C. Lee, D. Hudson, H. Birke-Sorensen, M. Depoorter, R. Dunn, S. Jeffery, F. Duteille, A. Bruhin, C. Caravaggi, M. Chariker, C. Dowsett, F. Ferreira, J. F. Martínez, G. Grudzien, S. Ichioka, R. Ingemansson, M. Malmsjo, P. Rome, S. Vig, N. Runkel, R. Martin, and J. Smith, "Evidence-based recommendations for the use of negative pressure wound therapy in traumatic wounds and reconstructive surgery: Steps towards an international consensus," Injury, vol. 42, pp. S1-S12, feb 2011. [Online]. Available: http: //dx.doi.org/10.1016/S0020-1383(11)00041-6

[14] A. Bruhin, F. Ferreira, M. Chariker, J. Smith, and N. Runkel, "Systematic review and evidence based recommendations for the use of negative pressure wound therapy in the open abdomen," International Journal of Surgery, vol. 12, no. 10, pp. 1105-1114, oct 2014. [Online]. Available: |http://dx.doi.org/10.1016/j.ijsu.2014.08.396 